in place to present to commissioners at an appropriate time so they could consider future commissioning.

The service has now been commissioned by the six Merseyside CCGs as a model of service delivery.

\section{P-201 AN INNOVATIVE MODEL OF CARE ENABLING A HOSPICE IN-PATIENT UNIT TO REACH MORE PEOPLE}

John Lansdell, Delyth Hughes, Lesley Spencer. Princess Alice Hospice, Esher, UK

\subsection{6/bmjspcare-2016-001245.222}

Background and context Within the UK, there is a general consensus that hospices should be extending their reach to care for more people, providing equity for those with a broader range of conditions. However, it is acknowledged that we are caring for people with increasingly complex needs and in reality there is a greater demand on beds. When viewed alongside nursing recruitment issues in hospices nationally, there is a need to review services to ensure we meet needs effectively and remain relevant.

What we are doing As a result of the changing context for palliative and end-of-life care, we have conducted a strategic review of our in-patient services. We plan to implement a new model of working, piloting two distinct wards each with a different nursing structure. One [specialist palliative care] ward will look after patients who have a high level of complex needs; this necessitates greater input from the multi-professional team, most notably the medical staff. The other will be a nurse-led [general palliative care] ward caring for a wider spectrum of patients who require less complex interventions. As well as caring for a greater number of patients, the model will also enable us to reach out to more people through education and training, offering clinical placements to our colleagues from non-specialist settings.

Evaluation The new way of working will be evaluated using an adapted action research approach at three-monthly intervals over a nine-month period with a final evaluation at 12 months.

Implications for practice It is anticipated that the new model of working will provide greater flexibility in the ways we can deploy our beds and resource, allowing us to care for more patients and 'reach more people' by sharing our knowledge, thereby improving the quality of care being delivered in other settings.

\section{P-202 NURSE CONSULTANT LEADERSHIP IN AN INTEGRATED HOSPICE SERVICE: EVALUATION OF IMPACT}

\footnotetext{
1,2Kate Heaps, 'Wendy Lethem, ${ }^{1,3,4,5,6,7}$ Steve Dewar. ${ }^{1}$ Greenwich and Bexley Community Hospice, London, UK; ${ }^{2}$ King's College London; ${ }^{3}$ Steve Dewar Associates Ltd; ${ }^{4}$ Together for Short Lives; ${ }^{5}$ St Christopher's Hospice; ${ }^{6}$ The Westcott Group Ltd; ${ }^{7}$ South East London Doctors Cooperative Ltd
}

\subsection{6/bmjspcare-2016-001245.223}

Background More people die in hospital than wish to do so and many are from an older age group. Without action this is likely to be a growing problem. By 2030, 44\% of all people dying will be over 85 years old.

In 2013 a business case supported the appointment of a hospice nurse consultant with a remit to work across hospice services, particularly those provided in hospital. The aim of the post was to reduce emergency admissions, length of stay and hospital deaths.

Key objectives were to: a. Increase use of the hospital palliative care team

b. Ensure further integration of services

c. Address the needs of the 'older old'

d. Connect palliative care expertise in and out of hospital

e. Develop strategies to meet growing demand.

Methods This evaluation draws together qualitative data and presents compelling evidence of impact against each objective. Establishing new systems and processes for referral to the hospital team and enabling rapid discharge has led to a significant reduction in deaths in non-critical hospital wards. The evaluation reviews the challenges and considers further developments.

Results Outcomes include:

- Referrals to the hospital team up $25 \%$ and hospital team visits up $89 \%$

- A $58 \%$ increase in referrals from the hospital team to the hospice community teams

- A 7\% decrease in deaths in non-critical hospital wards

- Almost 8 out of 10 patients discharge achieved within one week

- Increased reach to people with non-malignant disease.

Conclusion The initiative has had significant impact, far outweighing the investment required.

\section{P-203 SHARING THE LEARNING FROM AN INNOVATIVE PERINATAL HOSPICE SERVICE IN THE UK - AN INTEGRATIVE APPROACH TO ENABLING CHOICE}

Emma Bleasdale. Forget Me Not Children's Hospice, Huddersfield, UK

\subsection{6/bmjspcare-2016-001245.224}

Due to advances in imaging technology and routine screening many life-shortening fetal anomalies are detectable in the early antenatal period. Whilst this allows families the opportunity to make extremely difficult decisions surrounding the progression of the pregnancy, families report a vast lack of support when deciding to continue the pregnancy in the knowledge that their baby may die before birth, during birth or shortly after.

In March 2014 the hospice was successful in securing funding for a one-year perinatal in-reach project with its initial aim of working more closely with regional neonatal units and facilitating choice for families. Due to the success of the first year's project the hospice saw a vast increase in referrals and interest in its services, in particular the support that could be offered for an early detection, in utero baby with a life shortening anomaly.

In the twelve months prior to the project the hospice received one neonatal referral. In the past 24 months the hospice has supported 53 families having received referrals from 24 neonatal families and 29 families in the antenatal period. As a result of the continuing interest and rise in referrals into our perinatal service the hospice has been fortunate enough to have secured a further three years funding enabling further development of our specialist perinatal service.

Offering families the opportunity to access specialist perinatal palliative support from point of diagnosis (whether in the antenatal or neonatal period) has immense benefits to all involved. It allows for an individualised, consistent and integrative approach to care, ensuring that families are offered the choices of place of care, place of death and the services they feel most beneficial to them as as a family, resulting in a more compassionate and positive experience. 